\title{
Multi-period Dynamic Optimization Model for Plug-in Hybrid Electric Vehicles and Electric Vehicles Charging Service Station
}

\author{
Do Tuan Khanh ${ }^{1,2}$ and Feng Gao ${ }^{1}$ \\ ${ }^{1}$ Systems Engineering Institute, School of Electronic and Information Engineering, \\ $X i$ 'an Jiaotong University, Xi'an, China \\ ${ }^{2}$ Hung Yen University of Technology and Education, Hung Yen, Vietnam \\ khanhdotuan@gmail.com,2570067412@qq.com
}

\begin{abstract}
Due to their advantages over conventional vehicles and incentive policies of governments over the world, plug-in hybrid electric vehicles (PHEVs) and electric vehicles $(E V s)$ are rapidly penetrating in our society. Charging station network is the key infrastructure for the deployment of PHEVs and EVs in the future. In turn, charging service makes a new domain for doing business. In this paper, we propose a multi-period dynamic optimization model for charging service station. A dynamic nonlinear mathematical model for an objective function (i.e., maximum revenue) is built. A heuristic algorithm for narrowing search space is proposed to solve the problem of large curse of dimensionality. By using dynamic programming heuristic algorithm, the authors characterize the performance of optimal power allocation to PHEVs/EVs problem for PHEVs/EVs charging service station, and compare it with that of other methods. The simulation is completed using MATLAB. Results show that dynamic programming brings a means to improve operation efficiency in most of indicators.
\end{abstract}

Keywords: Plug-in hybrid Electric vehicles (PHEV), Electric vehicles (EV), charging service station, dynamic programming.

\section{Introduction}

The increasing in gasoline prices and environmental pollution has made Plug-in Hybrid Electric Vehicles (PHEVs) and Electric Vehicles (EVs) more effective in individual transportation selection. In fact, PHVEs and EVs are rapidly penetrating in our society. The sales of PHEVs have been growing by $80 \%$ each year since 2000 [1], and $10 \%$ of new vehicle sales are expected to be PHEVs by the year of 2015. The U.S. Department of Energy projects that approximately one million PHEVs will be on the road by 2015 and 425000 PHEVs will be sold in 2015 alone. At this penetration rate, PHEVs would account for $2.5 \%$ of all new vehicle sales in 2015 [2]. However, there still exist obstacles to the proliferation of using PHEV. The increasing number of PHEVs is expected to add $18 \%$ load to the existing power grids and this may impact to the grid reliability due to less predictable overloads [3]. In addition, the vehicles' relatively short driving range and their long battery charging times, as compared to gasoline vehicles, need to be improved. At this stage, the charging station availabilities are an essential factor for the successful deployment of PHEVs and EVs, along with their performance improvement. K. Morrow et al., [4] indicated that presently most of PHEVs and EVs are equipped with a Li-on lithium packets battery, whose capacity ranges from $10 \mathrm{~kW}$ to $40 \mathrm{~kW}$ and can be divided into level 1 and level 2. According to the electric car association, the popular rated voltage of battery packet is about $244 \mathrm{~V}$, some specific types of PHEVs and EVs can use a higher or lower voltage. In ref. [5], the authors simulated real-world parking deck scenarios and implemented four types of battery models. They then evaluated the optimal performance of the proposed large-scale PHEV/PEV charging algorithms under certain operating 
conditions. Their simulation results provided an overview of the impact of battery modeling on optimal performance.

Recently, scholars over the world have paid attention into the optimization of PHEVs and EVs charging station operation, especially the optimal power allocation for PHEV s/EVs. P. Richardson et al., [6] proposed a method for controlling the rate at which electric vehicles charge could lead to a better utilization of the existing networks. W. Su et al., [7-10] proposed computational intelligence-based centralized charging algorithms (e.g., Estimation Distribution Algorithm EDA, Particle Swarm Optimization and Gravitational Search Algorithm GSA) to achieve the optimal power allocation at a largescale public PHEVs/EVs charging facility. Meanwhile, heavy intermittent charging load of EVs will create bottlenecks in the supplying capacity and expose power system to severe security risks. Y. Cao et al., [11] proposed a method to control EV charging loads in response to time-of-use (TOU) price in a regulated market. They proposed a heuristic method to regulate between three conflict constraints and objectives, namely, TOU price, batteries' $S o C$, and charging cost. Results showed that their method could reduce the charging cost and flat the load curve if the peak and the valley time periods are partitioned appropriately. D. H. Banet et al., [12] investigated an operational framework for multiple PHEV charging stations. The objective of their study is maximizing the Quality of Service (QoS) of the charging station while ensuring the stability of the power grid. In their study, multi-queue system is used as the base tool for analysis. They also suggested that additional price control methods could be used to minimize the waiting time and improve the fairness in the service.

This paper investigates the multiple time periods dynamic optimization model for a typical charging station, which provides charging service to the demanded PHEVs/EVs. Herein, the arrivals of the vehicles in each period are assumed to be stochastic and follow Poisson distribution which is widely used in such cases. The objective of the study is to maximize the revenue obtained by the station owner who provides charging service over the planning horizon while ensuring the normal charge of each vehicle as well as the normal working of the power grid. Therein, a vehicle is said to be normally charged if the charging rate lies within the allowance limitation and the normal working of the power grid that the power grid will not be overloaded. Specifically, we divide time horizon into T periods. At the beginning of each period, we review the number of vehicles not fully charged from previous period and record the information of new arrivals; then, we make power allocation decision to each vehicle in such a way that objective function is maximized with regard to the previous decisions and not violating the constraints of the model.

The rest of this paper is organized as follow. Section two is modeling which provides the detail of the proposed optimization model. In section three, we provide the numerical analysis and discussions. In section four, we give the conclusions and future works.

\section{Problem Formulation}

\subsection{Objective Function}

In this section, we consider one period's revenue obtained by the station owner. In period $\mathrm{t}$, if she/he assigns the charging rate for vehicle $i$ as $S_{i, t}$, with unit price of energy $\left(C_{o s t}\right)$, then at the end of the period, the driver will receive a quantity of electricity as $P_{i, t}$, measured in $\mathrm{kW}$. The revenue that the station owner can receive from charging all vehicles in period $t$ can be expressed by the following equations.

$$
\begin{aligned}
& J_{t}=\sum_{i=1}^{N_{t}} w_{i, t} P_{i, t} C_{\cos t} \\
& w_{i, t}=f\left(\operatorname{Tr}_{i, t}, D_{i, t}\right)
\end{aligned}
$$


Where, $\mathrm{N}_{\mathrm{t}}$ is the number of vehicles in the charging station at time step $t ; P_{i, t}$ is the power allocated to $i$-th vehicle in time step $t$; $C_{o s t}$ is the unit price of energy; $\operatorname{Tr}_{i, t}$ is the remaining charging time of the $i$-th PHEV at time step $t ; D_{i, t}$ is the price difference between the real-time energy price and the price that a specific customer at the $i$-th PHEV charger is willing to pay at time step $t ; w_{i, t}$ is the charge weighting term of the $i$-th PHEV at time step $t$. The weighting term gives a response proportional to the attributes of a specific PHEV. For example, a particular vehicle has a lower initial state of charge $(S o C)$ and less remaining charging time. But, if the driver is willing to pay a higher price, the controller can consider giving priority to this PHEV charger to charge and allocate more power.

$$
w_{i, t} \propto\left[D_{i, t}, \frac{1}{T r_{i, t}}\right]
$$

Since the two terms $D_{i, t}, 1 / T r_{i, t}$ are not on the same scale, it is necessary to normalize all the terms to assign similar importance to them.

$$
\begin{gathered}
d_{i, t}=\frac{D_{i, t}-\operatorname{Min}\left[D_{i, t}\right]}{\operatorname{Max}\left[D_{i, t}\right]-\operatorname{Min}\left[D_{i, t}\right]} \\
t_{i, t}=\frac{1 / \operatorname{Tr}_{i, t}-\operatorname{Min}\left[1 / \operatorname{Tr}_{i, t}\right]}{\operatorname{Max}\left[1 / \operatorname{Tr}_{i, t}\right]-\operatorname{Min}\left[1 / \operatorname{Tr}_{i, t}\right]}
\end{gathered}
$$

The charging station operators may also have different interests and assign different importance factors to $t_{i, t}$, and $d_{i, t}$, depending on their own preferences. Thus:

$$
w_{i, t}=1+\alpha_{1} t_{i, t}+\alpha_{2} d_{i, t}
$$

In the optimal power allocation to PHEVs, $S o C$ is an important parameter of the battery. It describes the percentage of the battery energy that has been used and indicates how far a vehicle can be driven on it. $S o C$ is defined as the remaining capacity of a battery [13].

$$
\text { SoC=Remaining Capacity/Rated Capacity }
$$

If Ah capacity is used, the change of $S o C$ can be expressed as:

$$
\Delta S o C=S o C_{i, t+1}-S o C_{i, t}=\frac{1}{C a p_{i}} \int_{t}^{t+1} \mathrm{i}_{i, t} d t
$$

Where, $\operatorname{Cap}_{i}$ is the rated capacity of the battery.

The charging current is assumed to be constant over charging time interval $\Delta t$.

$$
\begin{gathered}
{\left[\operatorname{SoC}_{i, t+1}-\operatorname{SoC}_{i, t}\right] \operatorname{Cap}_{i}=I_{i, t} \Delta t} \\
\operatorname{SoC}_{i, t+1}-\operatorname{SoC}_{i, t}=\frac{I_{i, t} \Delta t}{\operatorname{Cap}_{i}}
\end{gathered}
$$

Where, $I_{i, t}$ is the charging current over $\Delta t$.

We assume that the battery charging is modeled as a capacitor circuit and which follows capacitor equation. $C_{b}$ is capacitance in Farads (Farad is the unit of capacitance).

$$
C_{\mathrm{b}} \frac{d U_{i}}{d t}=I_{i, t}
$$

Taking integration for both sides of above equation, we have:

$$
\int_{t}^{t+1} C_{\mathrm{b}} \frac{d U_{i}}{d t} d t=\int_{t}^{t+1} I_{i, t} d t
$$

Because $C_{b}, I_{i, t}$ are constant, so:

$$
C_{b}\left(U_{i, t+1}-U_{i, t}\right)=I_{i, t} \Delta t
$$




$$
U_{i, t+1}=\frac{I_{i, t}}{C_{\mathrm{b}}}+U_{i, t}
$$

From relation between current, voltage and power, we have:

$$
P_{i, t}=\frac{U_{i, t+1}+U_{i, t}}{2} I_{i, t}=\frac{I_{i, t} \Delta t+2 C_{\mathrm{b}} U_{i, t}}{2 C_{b}} I_{i, t}
$$

We assume that the voltage of the battery is linearly increased in charging process, and the charging current is constant over charging time interval $\Delta t$.

If we define charging rate $\left(S_{i, t}\right)$ are stated as percentages of rated capacity $\left(\mathrm{Cap}_{i}\right)$ as slow to fast...(i.e $0,0.1 C a p_{i}, 0.2 C_{a p}, 0.3 C_{a p}, 0.4 C_{a p}, 0.5 C_{i} a p_{i}$ ), then the relation between charging rate, charging current and rated capacity looks like:

$$
S_{i, t}=\frac{I_{i, t}}{\operatorname{Cap}_{i}} \Leftrightarrow I_{i, t}=S_{i, t} \operatorname{Cap}_{i}
$$

Substituting (16) into (15), we get:

$$
P_{i, t}=\frac{1}{2 C_{b}} S_{i, t}^{2} \operatorname{Cap}_{i}^{2} \Delta t+S_{i, t} \operatorname{Cap}_{i} U_{i, t}
$$

Substituting (17) into (1), we finally obtain the objective function expressed in term of charging rate:

$$
J_{\mathrm{t}}=\sum_{i=1}^{N_{t}} X_{i, t} w_{i, t}\left(\frac{1}{2 C_{b}} S_{i, t}^{2} \operatorname{Cap}_{i}^{2} \Delta t+S_{i, t} \operatorname{Cap}_{i} U_{i, t}\right) \frac{C_{o s t}}{1000}
$$

Where, $\Delta t$ is the period length of time in hour, $U_{i, t}$ is the voltage between two poles of the battery at the beginning of period and can be measured easily, $\mathrm{Cap}_{i}$ is the rated capacity of battery.

In the next section, we will model our problem as a dynamic optimization charging service operation with the goal of maximizing the revenue over the planning horizon follow Section 2.2.

\subsection{Dynamic Programming Formulation}

By changing the charging rate of each vehicle in each period of time, the owner of station can obtain different revenues for each period as well as revenues over entire the planning horizon. Literature showed that in such cases, dynamic programming is an effective tool for obtaining global optimum. Based on the characteristics of the problem and the information above, the backward recursion algorithm is applied to this optimal power allocation to $\mathrm{PHEV}$ /EVs. The general equation for a backward recursion is as follows.

If the planning horizon consists of $\mathrm{T}$ periods, Bellman equation of the problem, including the discounting of future return, is given as:

$$
\begin{aligned}
& V_{t}\left(U_{i, t}, w_{i, t}\right)=\max _{t \in T, S_{i, t}, i=1, \ldots, \ldots, N_{\mathrm{t}}}\left[\begin{array}{l}
J_{t}\left(S_{i, t}, U_{i, t}, w_{i, t}\right)+\beta J_{t+1}\left(U_{i, t+1}, w_{i, t+1}\right)+ \\
\beta^{2} J_{t+2}\left(U_{i, t+2}, w_{i, t+2}\right)+\cdots+\beta^{T-1} J_{t+(T-t)}\left(U_{i, t}, w_{i, t+(T-t)}\right)
\end{array}\right] \\
& V_{t}\left(U_{i, t}, w_{i, t}\right)=\max _{t \in T, S_{i, t}, i=1,2, \ldots, N_{\mathrm{t}}}\left\{\begin{array}{l}
J\left(S_{i, t}, U_{i, t}, w_{i, t}\right)+ \\
\left.\beta \max \left[\begin{array}{l}
J_{t-1}\left(U_{i, t+1}, w_{i, t+1}\right)+\beta^{2} J_{t+2}\left(U_{i, t+2}, w_{i, t+2}\right) \\
+\cdots+\beta^{T-1} J_{t+(T-t)}\left(U_{i, t+(T-t)}, w_{i, t+(T-t)}\right)
\end{array}\right]\right\}
\end{array}\right.
\end{aligned}
$$

We can rewrite (20) as: 


$$
\begin{aligned}
& V_{t}\left(\bar{U}_{t}, \bar{w}_{t}\right)=\max _{t \in T, S_{i, t}, i=1,2, \ldots, N_{\mathrm{t}}}\left\{\sum_{i=1}^{N_{t}} J_{t}\left(S_{i, t}, U_{i, t}, w_{i, t}\right)+\beta V_{t+1}\left(\bar{U}_{t+1}, \bar{w}_{t+1}\right)\right\} \\
& \bar{w}_{t}=\left[w_{1, t}, w_{2, t}, \cdots, w_{N_{t}, t}\right]^{T}, \quad \bar{U}_{t}=\left[U_{1, t}, U_{2, t}, \ldots, U_{N_{t}, t}\right]^{T}
\end{aligned}
$$

Where, $V_{t}\left(U_{i, t}, w_{i, t}\right)$ is the revenue to go and can be computed as backward recursion. Specifically, we start from the last period with the initial conditions. We traverse all available charging rate assignments in period $t$, calculating corresponding revenues, adding into "up to period" $t+1$ revenue to get "up to period" $t$ revenue, comparing alternatives and selecting the maximum one.

As a period is finished, the state of charge of $i-t h$ vehicle $\left(S o C_{i}\right)$ will be increased with an amount of $\Delta S o C_{i, t}$ calculated as:

$$
\Delta S o C_{i, t}=\frac{I_{i, t} \Delta t}{C a p_{i}}=S_{i, t} \Delta t
$$

\subsection{System Constrains}

Constraint (23) prevents overloading of the station, increasing its efficiency. The total power charged for the vehicles in each period must be less than or equal to the efficient power of the station.

$$
\sum_{i=1}^{N_{t}} P_{i, t} \leq P_{S_{-} \max }
$$

Where, $P_{i, t}$ is the power allocated to $i$-th vehicle at the time step $t ; P_{S_{-} \max }$ is the power available from the maximum power of the charging station.

Substituting (17) into (23), we get:

$$
\sum_{i=1}^{N_{t}}\left(\frac{1}{2 C_{b}} S_{i, t}^{2} \operatorname{Cap}_{i}^{2} \Delta t+S_{i, t} \operatorname{Cap}_{i} U_{i, t}\right) \leq P_{\mathrm{S}_{-} \max }
$$

Where, $U_{i, t}$ is the voltage at the two poles of the battery of $i$-th vehicle at the beginning of time step $t$. If the time step $t$ is the first time step, $U_{i, t}$ is equal to the initial voltage.

Constrain (25) prevents overcharging for each battery of every vehicle. The charging rate for these vehicles must be less than or equal to the maximum absorbable charging rate $\left(S_{i}^{\max }\right)$ of battery in that period. While, constraint (26) is used to minimize the over charged and energy waste when the value of $S o C$ is approximately equal to 1 .

$$
\begin{aligned}
& 0 \leq S_{i, t} \leq S_{i}^{\max } \\
& 0 \leq S_{i, t} \Delta t \leq 1-S o C_{i, t-1}
\end{aligned}
$$

\subsection{Heuristic Algorithm for Narrowing Search Space}

As presented above, dynamic programming is an effective tool for obtaining global optimum. However, the application of dynamic programming to solve power allocation to $\mathrm{PHEV} / \mathrm{EV}$ s problem is difficult because the number of PHEVs/EVs and the period of time are very large, leading to an extremely large curse of dimensionality. Therefore, in this section, a heuristic algorithm for narrowing search space is proposed. The algorithm can be called as customer's priority rating according to weighting term $\left(\mathrm{w}_{\mathrm{i}, \mathrm{t}}\right)$ value. The heuristic algorithm differentiates the customer according to their needs, assigning them with an appropriate charging rate. However, the maximization of total revenue of the charging station should is ensured. The heuristic algorithm for narrowing search space is formulated as follows. 
The weighting term of all PHEVs/EVs at each time step are sorted. Meanwhile, the number of PHEVs/EVs with high priority at the each time step to be charged is computed using (27) and (28):

$$
\mathrm{N}_{\mathrm{ch,t}}=\frac{P_{S_{-} \max }}{P_{\mathrm{i}, \mathrm{t}}^{\max }}+\mathrm{n}_{0}
$$

Where; $\mathrm{P}_{\mathrm{i}, \mathrm{t}}{ }^{\max }$ is calculated by (17). Where, $\mathrm{S}_{\mathrm{i}, \mathrm{t}}{ }^{\max } ; N_{c h, t}$ is the total number of vehicles to be charged at time step $\mathrm{t} ; n_{0}$ is a binary variable.

$$
\mathrm{n}_{0}= \begin{cases}0 & \sum_{1}^{N_{c h, t}} \mathrm{P}_{\mathrm{i}, \mathrm{t}}^{\max }=P_{S_{-} \max } \\ 1 & \sum_{1}^{N_{c h, t}} \mathrm{P}_{\mathrm{i}, \mathrm{t}}^{\max } \neq P_{S_{-} \max }\end{cases}
$$

We consider there exists a larger total revenue of the charging station when transferring an amount capacity $\Delta \mathrm{P}$ from $l$-th vehicle with largest weighting term to $k$-th vehicle with less weighting at time step $\mathrm{t}\left(l=\left[1,2, \ldots, \mathrm{N}_{\mathrm{ch}, \mathrm{t}}\right] ; k=\left[\mathrm{N}_{\mathrm{ch}, \mathrm{t}}+1, \mathrm{~N}_{\mathrm{ch}, \mathrm{t}}+2, \ldots, \mathrm{N}_{\mathrm{t}}\right]\right)$. And at the time step $t+n(\mathrm{n}=[1,2,3, \ldots, \mathrm{T}-\mathrm{t}])$, the $l$-th vehicles will receive an amount of capacity $\Delta P$ and $k$-th vehicle. This case exists if and only if the condition (29) is satisfied.

$$
\sum_{1}^{T} \mathrm{~J}_{t}<\sum_{1}^{T} \overline{\mathbf{J}}_{t}
$$

$\sum_{1}^{T} \mathrm{~J}_{t} \sum_{\text {Whd }}^{T} \overline{\mathrm{J}}_{t}$ is total revenue of charging station before
amount capacity $\Delta P$.
Where,
\[ \sum_{1}^{T} \mathrm{~J}_{t}=\sum_{1}^{\mathrm{N}_{1}} \mathrm{~J}_{1}+\sum_{1}^{\mathrm{N}_{2}} \mathrm{~J}_{2}+\cdots+\sum_{1}^{\mathrm{N}_{t}} \mathrm{~J}_{t}+\sum_{1}^{\mathrm{N}_{t+1}} \mathrm{~J}_{t+1}+\cdots+\sum_{1}^{\mathrm{N}_{t+n}} \mathrm{~J}_{t+n}+\cdots+\sum_{1}^{\mathrm{N}_{\mathrm{T}}} \mathrm{J}_{T} \]

$$
\sum_{1}^{T} \overline{\mathbf{J}}_{t}=\sum_{1}^{\mathrm{N}_{1}} \overline{\mathbf{J}}_{1}+\sum_{1}^{\mathrm{N}_{2}} \overline{\mathbf{J}}_{2}+\cdots+\sum_{1}^{\mathrm{N}_{t}} \overline{\mathbf{J}}_{t}+\sum_{1}^{\mathrm{N}_{t+1}} \overline{\mathbf{J}}_{t+1}+\cdots+\sum_{1}^{\mathrm{N}_{t+n}} \overline{\mathbf{J}}_{t+n}+\cdots+\sum_{1}^{\mathrm{N}_{\mathrm{T}}} \overline{\mathbf{J}}_{T}
$$
$\mathrm{T}$.

Where, $N_{1}, N_{2}, N_{3} \ldots, N_{T}$ are the number of the vehicles at the time step 1 to the time step The formulas (30) and (31) show that only $\sum_{1}^{N_{t}} \overline{\mathbf{J}}_{t}$ and $\sum_{1}^{N_{t+n}} \overline{\mathbf{J}}_{t+n}$ are changed, while the others remain unchanged.

The (4-29) exists if and only if the following condition is satisfied:

$$
\sum_{1}^{\mathrm{N}_{t}} \mathrm{~J}_{t}+\sum_{1}^{\mathrm{N}_{t+n}} \mathrm{~J}_{t+n}<\sum_{1}^{\mathrm{N}_{t}} \overline{\mathrm{J}}_{t}+\sum_{1}^{\mathrm{N}_{t+n}} \overline{\mathrm{J}_{t+n}}
$$

Where,

$$
\begin{aligned}
& \sum_{1}^{\mathrm{N}_{t}} \mathrm{~J}_{t}=\mathrm{J}_{1, t}+\mathrm{J}_{2, t}+\cdots+\mathrm{J}_{k, t}+\mathrm{J}_{l, t}+\cdots+\mathrm{J}_{N_{t}, t} \\
& \sum_{1}^{\mathrm{N}_{t+n}} \mathrm{~J}_{t}=\mathrm{J}_{1, t+n}+\mathrm{J}_{2, t+n}+\cdots+\mathrm{J}_{k, t+n}+\mathrm{J}_{l, t+n}+\cdots+\mathrm{J}_{N_{t}, t+n} \\
& \sum_{1}^{\mathrm{N}_{t}} \overline{\mathbf{J}}_{t}=\overline{\mathbf{J}}_{1, t}+\overline{\mathbf{J}}_{2, t}+\cdots+\overline{\mathbf{J}}_{k, t}+\overline{\mathbf{J}}_{l, t}+\cdots+\overline{\mathbf{J}}_{N_{t}, t}
\end{aligned}
$$




$$
\sum_{1}^{N_{t+n}} \overline{\mathbf{J}}_{t}=\overline{\mathbf{J}}_{1, t+n}+\overline{\mathbf{J}}_{2, t+n}+\cdots+\overline{\mathbf{J}}_{k, t+n}+\overline{\mathbf{J}}_{l, t+n}+\cdots+\overline{\mathbf{J}}_{\mathrm{N}_{t}, t+n}
$$

It can be seen from (33) to (36) that only $\overline{\mathbf{J}}_{k, t+n}+\overline{\mathbf{J}}_{l, t+n}$ is changed, the others are not changed.

The (32) exists if and only if the following condition is satisfied.

$$
\mathbf{J}_{k, t}+\mathbf{J}_{l, t}+\mathbf{J}_{k, t+n}+\mathbf{J}_{l, t+n}<\overline{\mathbf{J}}_{k, t}+\overline{\mathbf{J}}_{l, t}+\overline{\mathbf{J}}_{k, t+n}+\overline{\mathbf{J}}_{l, t+n}
$$

Where,

$$
\left\{\begin{array}{l}
\mathrm{J}_{k, t}=w_{k, t} \mathrm{P}_{k, t} C_{\cos t} \\
\mathbf{J}_{l, t}=w_{l, t}\left(\mathrm{P}_{l, t}^{\prime}+\Delta \mathrm{P}\right) C_{\cos t} \\
\mathbf{J}_{k, t+n}=w_{k, t+n}\left(\mathrm{P}_{k, t+n}^{\prime}+\Delta \mathrm{P}\right) C_{\cos t} \\
\mathbf{J}_{l, t+n}=w_{l, t+n} \mathrm{P}_{l, \mathrm{t}+n} C_{\cos t} \\
\overline{\mathbf{J}}_{k, t}=w_{k, t}\left(\mathrm{P}_{k, t}+\Delta \mathrm{P}\right) C_{\cos t} \\
\overline{\mathbf{J}}_{l, t}=w_{l, t} \mathrm{P}_{l, t}^{\prime} C_{\cos t} \\
\overline{\mathbf{J}}_{k, t+n}=w_{k, t+n} \mathrm{P}_{k, t+n}^{\prime} C_{\cos t} \\
\overline{\mathbf{J}}_{l, t+n}=w_{l, t+n}\left(\mathrm{P}_{l, t+n}+\Delta \mathrm{P}\right) C_{\cos t}
\end{array}\right.
$$

Where, $P_{k, t}, P_{l, t}\left(P_{l, t}=P_{l, t}^{\prime}+\Delta P\right)$ are the amount of capacity that $k$-th vehicle and $l$-th vehicle obtain at the time step t; $P_{l, t+n}, P_{k, t+n}\left(P_{k, t+n}=P_{k, t+n}^{\prime}+\Delta P\right)$ are the amount of capacity that $k$-th vehicle and $l$-th vehicle obtain at the time step $t+n$;

Substituting (38) into (37), we get:

$$
\begin{aligned}
& w_{k, t} \mathrm{P}_{k, t} C_{\text {cost }}+w_{l, t}\left(\mathrm{P}_{l, t}^{\prime}+\Delta \mathrm{P}\right) C_{\text {cos } t}+w_{k, t+n}\left(\mathrm{P}_{k, t}^{\prime}+\Delta \mathrm{P}\right) C_{\text {cost }}+w_{l, t+n} \mathrm{P}_{l, t+n} C_{\text {cos } t}< \\
& w_{k, t}\left(\mathrm{P}_{k, t}+\Delta \mathrm{P}\right) C_{\text {cost }}+w_{l, t} \mathrm{P}_{l, t}^{\prime} C_{\text {cost }}+w_{k, t+n} \mathrm{P}_{k, t}^{\prime} C_{c o s t}+w_{l, t+n}\left(\mathrm{P}_{l, t+n}+\Delta \mathrm{P}\right) C_{\text {cost }}
\end{aligned}
$$

Implementing some modifications (39), we obtain.

$$
\begin{aligned}
& w_{k, t}+w_{l, t+n}>w_{l, t}+w_{k, t+n} \\
\Leftrightarrow & w_{l, t+n}-w_{k, t+n}>w_{l, t}-w_{k, t}
\end{aligned}
$$

From the analysis above, we can see that the condition (29) exists only if the constrain (41) is satisfied.

However, we can see the $l$-th vehicle only transfer an amount of capacity $\Delta P$ for $k$-th vehicle if the following constrain (42) is satisfied:

$$
\operatorname{Tr}_{l, t} * S_{l, t}^{\max }>1-S S o C_{l}
$$

Where, $T_{r, l, t}$ is the remaining charging time of the $l$-th vehicle at the time step $t ; S_{l, t}{ }^{\max }$ is the maximum charging rate; $S S o C_{l}$ is the initial state of charge of the $l$-th vehicle.

In order to prove that the $l$-th vehicle only transfer an amount capacity $\Delta P$ for $k$-th vehicle if the following constrain (42) is satisfied, we conduct a little test. In this test, we assume that the remaining charging time of $l$-th vehicle is one hour (est. 4 time steps), and the initial state of charge $S S o C_{l}$ is equal to 0.3. In addition the $l$-th vehicle has the highest priority corresponding to level charging rate is maximum charging rate $\left(\mathrm{S}_{\mathrm{i}, \mathrm{t}}{ }^{\max }\right)$. In this case, if an amount capacity $\Delta \mathrm{P}$ is transferred from $l$-th vehicle to $k$-th vehicle (ex. $\Delta \mathrm{P}=0.2$ ), after one hour the $l$-th vehicle will obtain an amount capacity as shown inTable 1: 
Table 4-1

\begin{tabular}{lllll}
\hline Time step & Step 1 & Step 2 & Step 3 & Step 4 \\
\hline Charging rate & $\mathrm{S}_{\mathrm{i}, \mathrm{t}}{ }^{{ }^{\max }}-\Delta \mathrm{P}$ & $\mathrm{S}_{\mathrm{i}, \mathrm{t}}{ }^{\max }$ & $\mathrm{S}_{\mathrm{i}, \mathrm{t}}{ }^{\max }$ & $\mathrm{S}_{\mathrm{i}, \mathrm{t}}{ }^{\max }$ \\
\hline
\end{tabular}

From Table 4-1, we can see that in the time steps from 2 to 4 , the l-th vehicle cannot receive an amount capacity $\Delta P$ because it is charged with the maximum charging rate in these time steps. This implies that constrain (41) and condition (29) do not exist.

Generally, the formula (41) and (42) can be rewritten as:

$$
\begin{aligned}
& w_{i, t+n}-w_{j, t+n}>w_{i, t}-w_{j, t} \\
& \operatorname{Tr}_{i, t} * S_{i, t}^{\max }>1-\operatorname{SSoC} C_{i}
\end{aligned}
$$

Where, $i=\left[1,2, \ldots, \mathrm{N}_{\mathrm{ch}, \mathrm{t}}\right]$ and $j=\left[\mathrm{N}_{\mathrm{ch}, \mathrm{t}}+1, \mathrm{~N}_{\mathrm{ch}, \mathrm{t}}+1, \ldots, \mathrm{N}_{\mathrm{t}}\right]$

Therefore, transferring an amount of capacity $\Delta P$ from the large-weighting term vehicle to the less-weighting term vehicle happens only if the two constraints (43) and (44) are satisfied.

If the transferring an amount capacity $\Delta P$ do not happens, then the customer with a largest weighting term $\left(w_{i, t}\right)$ value will be assigned with a maximum charging rate. And the next vehicles with the largest weighting term will be performed similarly with a charging rate equal to or less than the maximum charging rate until the capacity of charging station equal to 0 .

Customers, who stay in the station for more than one period, will be updated with a new weighting term $w_{i, t}$ after each time step. In brief, the algorithm includes some steps below.

Step 1: At the beginning of each time step, the value of willing to pay and the remaining charging time of each customer are recorded.

Step 2: The willing to pay, the remaining charging time, and the weighting term $w_{i, t}$ of all vehicles at each time step are calculated.

Step 3: The customers are sorted into a list according to the priority (weighting term) in a descending order.

Step 4: This step is recursion to generate assignments. If the two constraints (43) and (44) are satisfied, then an amount of capacity $\Delta \mathrm{P}$ will be transferred from the largestweighting term vehicle to the less-weighting term vehicle. If two constraints (43) and (44) are not satisfied, then the vehicle with largest weighting term will first be assigned with maximum charging rate. And the next largest-weighting term customer will be performed similarly with a charging rate equal to or less than maximum charging rate until the capacity of charging station equal to 0 .

Applying this algorithm to our study not only improves the fairness and revenue for the station owner but also reduces the search space, especially for the problem with a large number of charging rate steps and number of priority levels. Applying the dynamic programming heuristic algorithm for narrowing search space to solving optimal power allocation to EV problem is presented in Figure 1. 


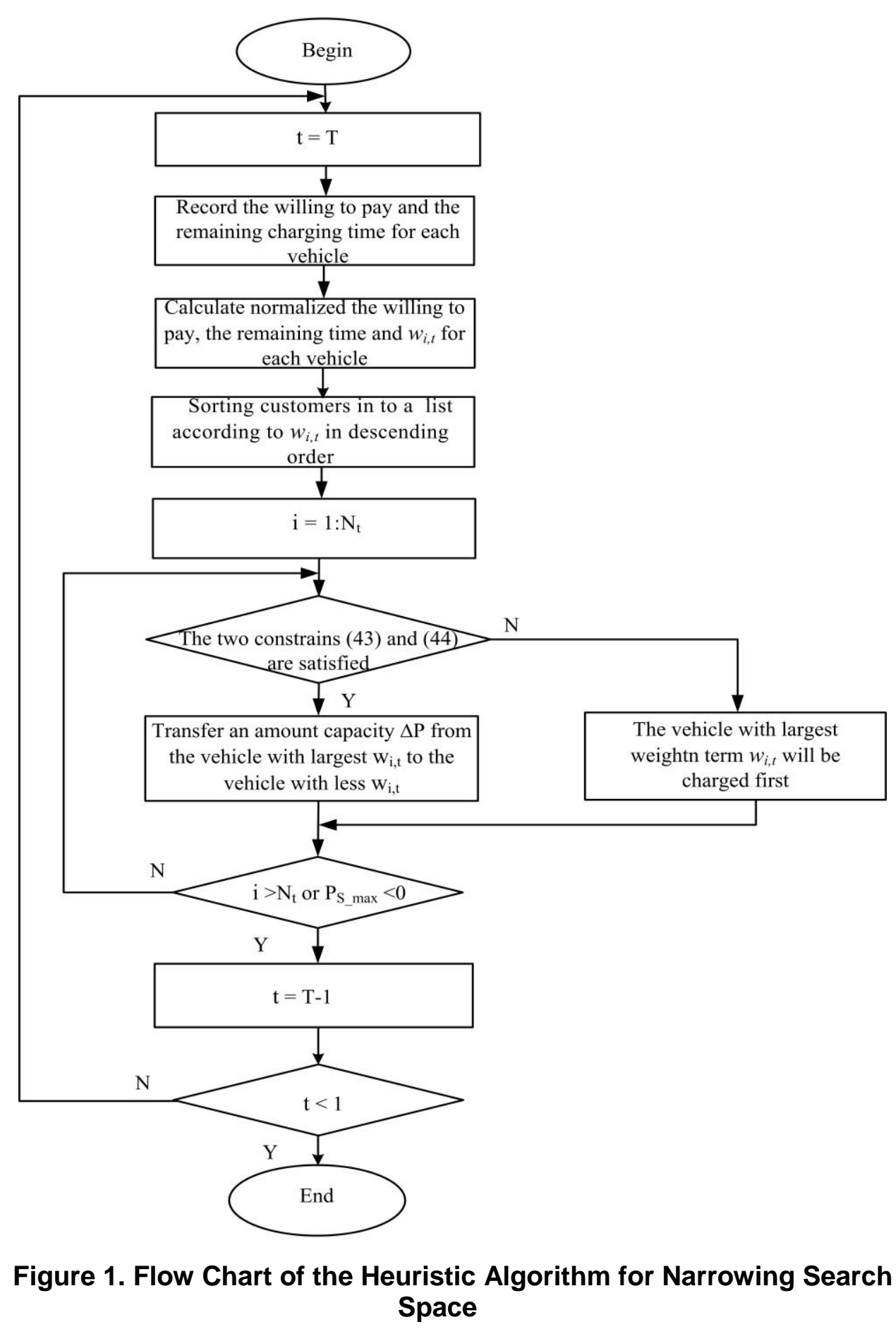

\section{Simulation Results and Analysis}

In this section, the authors analyze the simulation results of the dynamic programming heuristic algorithm (DPH), and compare it with other methods such as largest weighting term first (LWF), equally allocation method (EAM), and first come first serve strategy (FCFS).

\subsection{Largest Weighting Term First Strategy}

In the largest weighting term first strategy (LWF), the total vehicles charged in each time step includes the vehicles left from the previous time steps and just arrived. At each 
time step, the weighting term of all vehicles will be updated and evaluated and the vehicle with largest weighting term $\left(w_{i, t}\right)$ will be charged first.

\subsection{Equally Allocation Method}

Equally allocation method (EAM), the total capacity of the charging station at each time step will be equally allocated among the vehicles waiting at the station, with the constraint $P_{i}(t) \leq P_{i}^{m a x}(t)$. The equal allocation method can be expressed as follows.

$$
\begin{aligned}
& \mathrm{P}_{i}(t)=\frac{P_{\mathrm{S}-\max }}{N(t)} \\
& \text { st. } \quad P_{i}(t) \leq P_{i}^{\max }(\mathrm{t})
\end{aligned}
$$

Where $P_{i}(t)$ is power allocated to $i$-th vehicle at the time step $t, N(t)$ is the number of the vehicles at the time step t, $P_{i}^{\max }(t)$ is the maximum power that vehicles are charged at each time step, and $\mathrm{P}_{\mathrm{S}_{-} \max }$ is the maximum power of charging station

\subsection{First Come First Serve Strategy}

In the first come first serve strategy (FCFS), the total vehicles charged in each time step includes the vehicles left from the previous time steps and just arrived. The vehicle comes first will be served first and will only leave when it is fully charged or the customer requiring time is out (remaining charging time equal to zero). The number of total vehicles charged in each time step is the division of total power of station and the maximum power $\left(P_{i}^{\max }\right)$ that can be absorbed by a specific vehicle plus the binary variable $n_{0}\left(n_{0}\right.$ is 1 as the remainder of the division is nonzero and $n_{0}$ is 0 as the as the remainder of the division is zero). The FCFS strategy is demonstrated in the following formulas:

$$
\begin{aligned}
& \mathrm{N}_{\mathrm{ch}}(\mathrm{t})=\frac{P_{\mathrm{S}-\max }}{\mathrm{P}_{\mathrm{i}}^{\max }(t)}+\mathrm{n}_{0} \\
& \mathrm{n}_{0}= \begin{cases}0 & \sum \mathrm{P}_{\mathrm{i}}^{\max }(t)=P_{S-\max } \\
1 & \sum \mathrm{P}_{\mathrm{i}}^{\max }(t)<P_{\mathrm{S}-\max }\end{cases}
\end{aligned}
$$

Where, $N_{c h}(t)$ is the total number of vehicles to be charged at time step t, and $n_{0}$ is a binary variable.

\subsection{Data and Results Analysis}

Due to lack of real market data, some of the parameters are estimated or simulated according to published work and public data [6-8]. Battery chargers fall into three categories based on voltage and power level. Level 2 is typically described as the primary or standard method for both private and public charging, and specifies a single-phase branch circuit with typical voltage of 240 VAC. In this paper, all the battery chargers are assumed to be Level 2 and the maximum PHEV charging rate limit is $S_{i, t}{ }^{m a x}=0.5 C_{a p}$. The station capacity in the test is $P_{S_{-} \max }=187 \mathrm{~kW}$.

In this study, the number of vehicles $m_{t}$ arriving to station at each time step to request charging service is stochastic. In such a case, the arrival times are assumed to follow Poisson distribution with the mean parameter lambda $\lambda$ and the probability mass function of $m_{t}$ is given by:

$$
f=\left(m_{t} \mid \lambda\right)=\frac{\lambda^{\mathrm{m}_{t}} e^{-\lambda}}{\mathrm{m}_{\mathrm{t}} !}
$$

Where, $m_{t}$ is the number of PHEVs arriving to the charging station each time step; e is Euler's number $(\mathrm{e}=2.71828 \ldots)$. 
Moreover, the initial $S o C$ of vehicles are assumed to follow log-normal distribution with mean $\mu$ and standard deviation $\sigma$. Therefore, the probability of initial $S o C$ can be computed as (47).

$$
f\left(S o C_{i} \mid \mu, \quad \sigma\right)=\frac{1}{S o C_{i} \sqrt{2 \pi \sigma^{2}}} e^{-\frac{\left(\ln S o C_{i}-\mu\right)^{2}}{2 \sigma^{2}}}
$$

In this test, we considered 96 time steps corresponding to 24 hours; the sample time was set for each time step is 15 minutes. The remaining charge time of the vehicle arriving to station at each time step was defined as a continuous random number between 1 and 8 hours. The price that the customer was willing to pay for electricity was defined as a continuous random number between $\$ 1$ and $1.5 \$$. The battery capacity was assumed to be identical for all vehicles. Weighting term $w_{i}(t)$ was the summation of three terms normalized attributes, and followed the relation:

$$
w_{i, t}=1+\alpha_{1} t_{i, t}+\alpha_{2} d_{i, t}
$$

Where, $\alpha_{1}, \alpha_{2}$, are the coefficient factors and used to evaluate the importance of each attribute of the weighting term. Their values depend on station characteristics, demand type, etc. In this paper, we assumes that $\alpha_{1}=0.2 ; \alpha_{2}=0.8$;

\subsubsection{Cumulative Revenue}

Figure 2 shows that the cumulative revenue of the DPH and the LWF methods at each time step are always greater than that of the other two methods. This is because the allocation power strategy to PHEVs/EVs of each method is different. In the LWF method, the vehicle with largest weighting term $\left(w_{i, t}\right)$ will be prioritized before being charged with maximum charging rate. This means that the total of revenue of the LWF method at the each time step is maximizing, and it is called local optimal power allocation strategy.

In order to maximize the total revenue of the charging station and guarantee that the vehicle with large $w_{i, t}$ is also obtained maximum capacity when leaving station, in the DPH method, an amount capacity $\Delta P$ is transferred from the vehicle with largest $w_{i, t}$ to the vehicle with less $w_{i, t}$, if two constrains (43) and (44) are satisfied. If the two constraints are not satisfied, then the vehicle with largest weighting term $\left(w_{i, t}\right)$ will be charged first with a maximum charging rate.

From the analysis above, we can see that in some time steps the cumulative revenue of the DPH method is less than that of the LWF method. Figure 2 also shows that in the last time step the cumulative revenue of the DPH method is greater than that of the LWF method, which implies that the goal of maximizing revenue over the planning horizon is satisfied. The DPH method is called global optimal strategy.

In the EAM method, all of the vehicles are charged with a similar charging rate according to (45). Thus, the unit price of energy $C_{\text {cost }}$ is identical at the each time step for all vehicles. In addition, for the vehicles with $S o C$ approximately equal to 1 , the equally allocation power strategy can cause capacity waste. Especial, the FCFS with power allocation strategy according to (46) can cause more capacity waste than the EAM method. Figure 2 shows that the total revenue of the FCFS is always less than the total revenue of the other method.

Table 2 shows the total revenue of the charging station in 24 hours of the each method. It indicates that DPH method can increase the total revenue of the charging station up 2\% as compared to the LFW method, increasing $11.4 \%$ as compared to the EAM method, and increasing $21.4 \%$ compared to the FCFS method. 
Table 2. Cumulative Revenue at the $96^{\text {th }}$ Time Step

\begin{tabular}{|c|c|}
\hline Power allocation methods & Total revenues of the charging station \\
\hline DPH & 20899.414 \\
\hline LWF & 20327.862 \\
\hline EAM & 18765.942 \\
\hline FCFS & 17210.375 \\
\hline
\end{tabular}

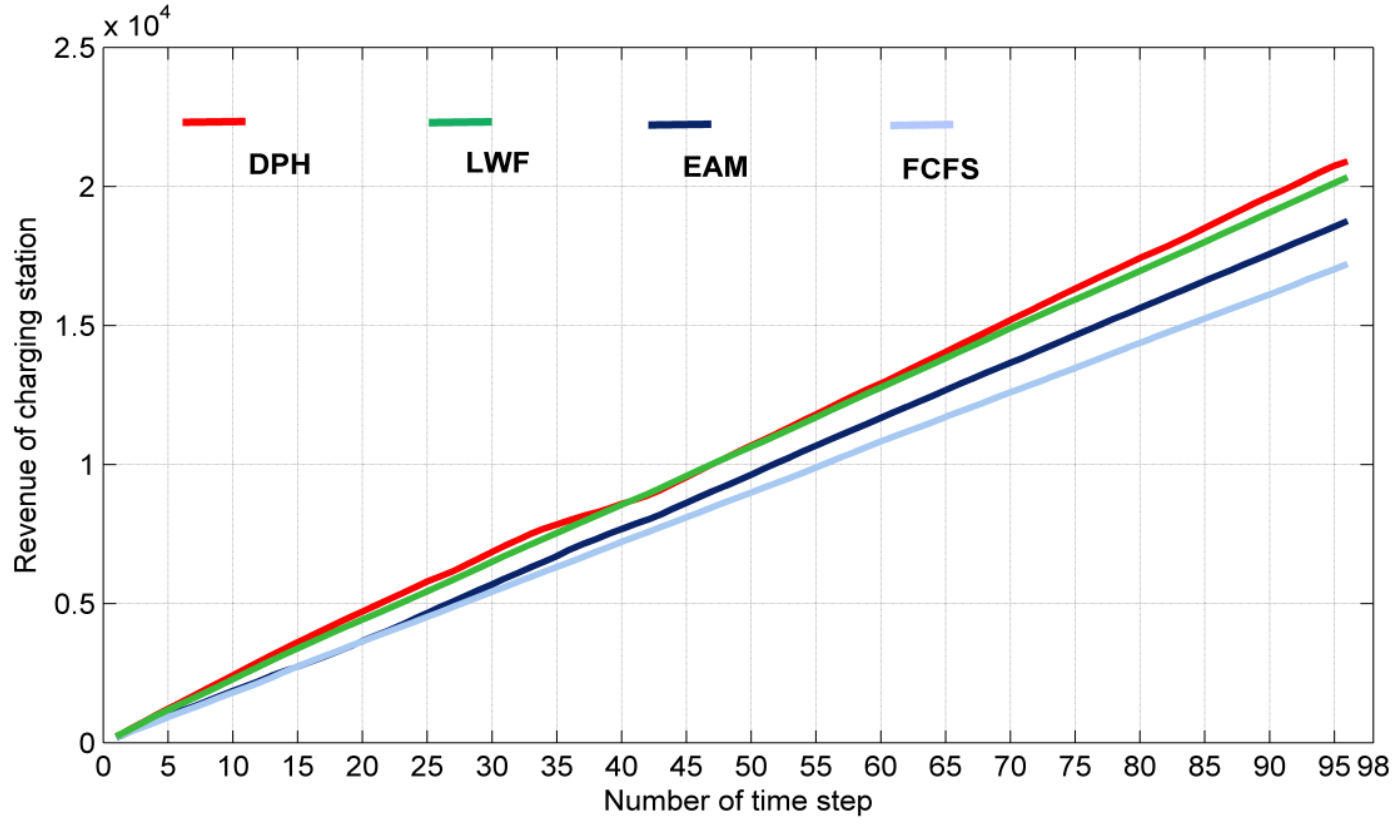

Figure 2. Cumulative Revenue over Planning Horizon

\subsubsection{Departure SoC of EVs}

In this section, we consider 413 vehicles out of the total 500 vehicles in the charging station that ran out of time in the charging station. Then, we compare the SoC of the vehicles of the power allocation method obtained during the charging time at the charging station. Figure 3 shows that the initial $\mathrm{SoC}$ of the vehicles are generated randomly according to log-normal distribution. Meanwhile, figures 4 (a), (b), (c) and (d) illustrate the departure $\mathrm{SoC}$ of the vehicles when leaving the station when using different power allocation methods.

Figure 4 shows that the departure SoC of the all vehicles in the DPH method and the LWF method are greater than or equal to 0.7. This means that a large number of vehicles are fully charged (357 over 413 vehicles leave the station). Meanwhile, in the EAM method, the number of vehicles with the departure $\mathrm{SoC}$ of greater than or equal to 0.7 is 365 vehicles out of 413 vehicles leaving the charging station. In the FCFS method the number vehicles with the departure SoC greater than or equal to 0.7 are 343 out of 413 vehicles leaving the charging station. Besides, we can see in the FCFS method and the EAM methods, there is a large number of vehicles with SoC smaller than 0.5. Even in the FCFS method, the number of vehicles with SoC smaller than 0.4 are not small. This indicates that those vehicles are not allocated power or allocated with a small amount power during charging time at the station.

The difference in $S o C$ obtained in the four methods could be explained as follows. In EAM method, in each time period, the total power of the station is distributed equally to the vehicles in the station as long as $S_{i, t}<S_{i, t}^{{ }^{m a x}}\left(P_{i}(t) \leq P_{i}^{{ }^{\max }}(t)\right)$. The more the number of vehicle in the station, the less the power is distributed for each vehicle in a period of time. 
Thus, the vehicles staying for a long time in the station could be charged fully $(S o C=1)$ and the ones staying for a shot time are distributed a small amount of power and could only be partially charged. At the mean time, in FCFS method, a vehicle that comes first is charged first with a highest charging rate $S_{i, t}{ }^{\max }\left(P_{i}(t)=P_{i}^{{ }^{\max }}(t)\right)$ until it is fully charged. This results into a severe charging problem for the vehicle that comes later and gets a short charging time since it can only be charged when the vehicle that came before is fully charged. In other word, a vehicle with a short charging time is distributed a very small amount of power, even when it leaves, it is not charged as shown in Figure 4 (c).

In the DPH method and the LWF method, each vehicle is assigned with a weighting term $w_{i, t}$, which depends on the parameters such as remaining charging time $\operatorname{Tr}_{i, t}$ and especially cost willing to pay $D_{i, t}$. In the DPH method and the LWF method, according to the value of weighting term $w_{i, t}$, at the each time step, the vehicle with largest $w_{i, t}$ will be charged first. In addition, to maximize the total revenue of charging station and the vehicle with largest $w_{i, t}$ is obtained maximum capacity level as leaving the station. An amount capacity $\Delta P$ is transferred from the vehicle with a largest $w_{i, t}$ to the vehicle with less $w_{i, t}$ based on two constraints (43) and (44). The value of the remaining charging time $\operatorname{Tr}_{i, t}$ is changed at each period time, leading to the change of the weighting term of each vehicle. Hence, most of the vehicles are charged with a reasonable amount of power after leaving the station.

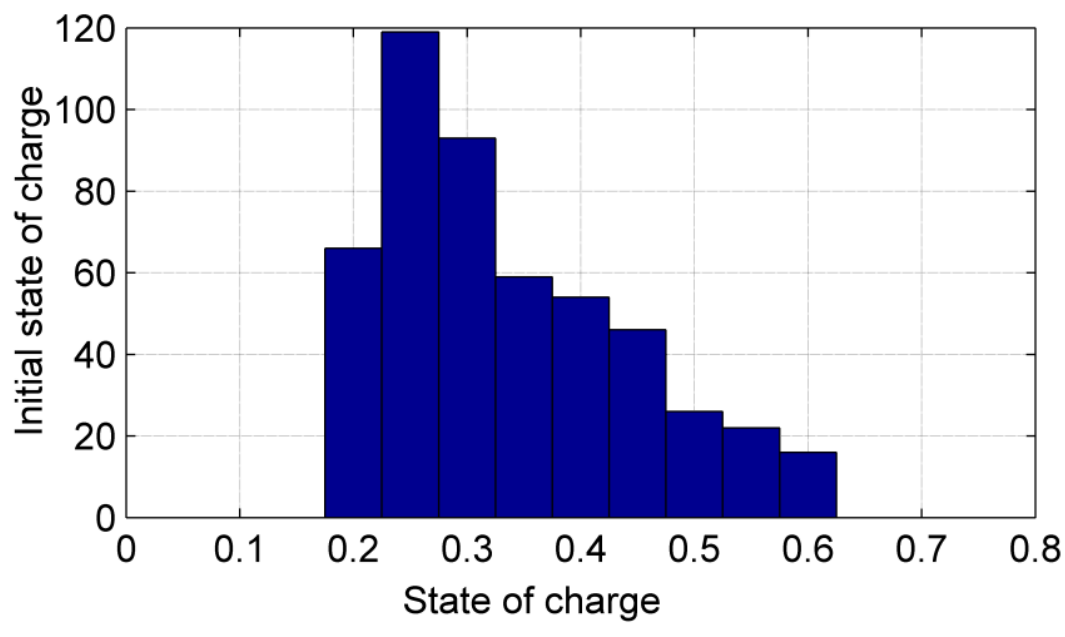

Figure 3. Initial SoC for 500 EVs Case 


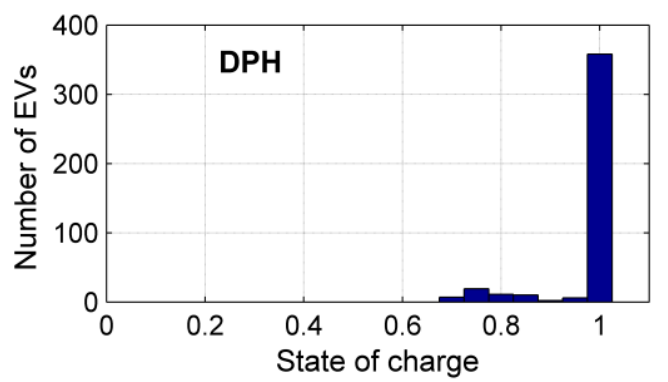

(a)

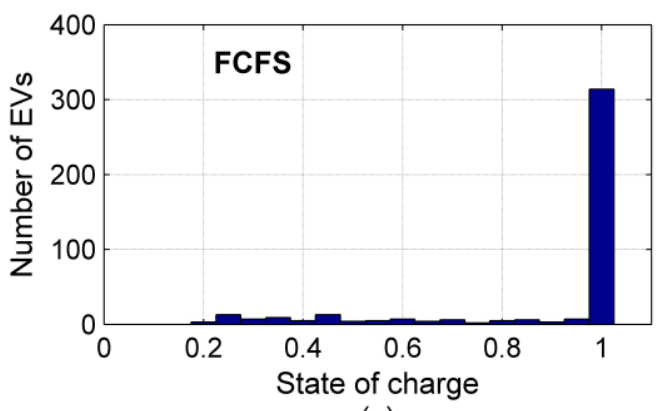

(c)

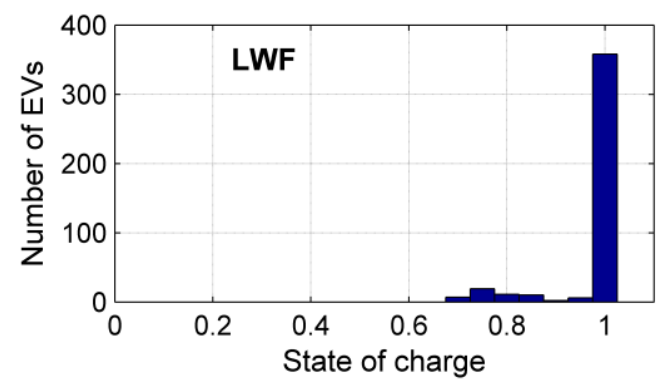

(b)

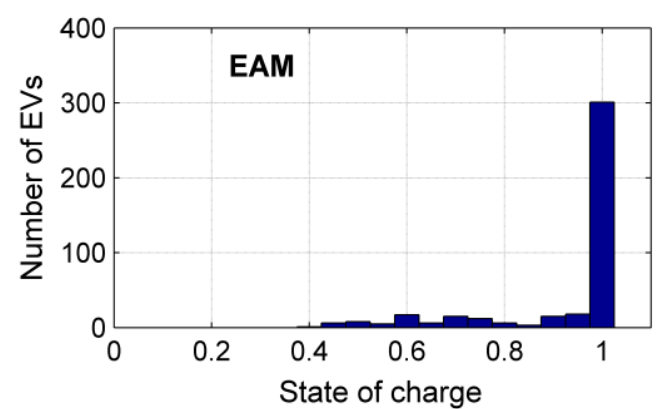

(d)

Figure 4. Departure SoC of 413 EVs for 500 EVs case; (a) Departure SoC of DPH method; (b) Departure SoC of LWF method; (c) Departure SoC of FCFS method; (d) Departure SoC of EAM method

\subsubsection{Analysis Satisfying Customer Demand}

There are some different properties of the power allocation system in the charging station as compared to the practical serving one. For example, there are a number of customers who want to leave the station for a long time ( 8 hours at work) and there are some who want to charge quickly and willing to pay more for the priority. Hence, a suitable and flexible charging strategy is necessary to satisfy all the customers.

In order to assess the ability to satisfy the demand of customer, we consider 15 vehicles from the $70^{\text {th }}$ vehicle to the $84^{\text {th }}$ vehicle via the parameters such as: the initial state of charge $(S S o C)$, the initial remaining charging time $T r_{i, t}$ and the departure $S o C$. Figure 5 shows that most of the vehicles with a long charging time are fully charged when leaving the charging station, specifically the vehicles $73^{\text {th }}, 75^{\text {th }}, 81^{\text {th }}$ and $83^{\text {th }}$. This means that the power allocation methods satisfied the customer's demand. But for the vehicles with a short remaining charging time, for example the vehicles with the remaining charging time only two hours or one hour, as can be seen in the EAM method and the LWF method, the departure $S o C$ of these vehicles are very small, some departure SoC of the vehicles are equal to initial state of charge $(S S o C)$, meaning that those vehicles are only allocated a small amount capacity or even not allocated at all. Meanwhile, in the DPH method and the LWF method, the vehicles with remaining charging time equal to two hours are fully charged, while the vehicles with remaining charging time equal to one hour are also obtained departure $\mathrm{SoC}$ greater than 0.7 (SoC> 0.7). Thereby, we can confirm that the DPH method and the LWF method satisfy customer demand. 

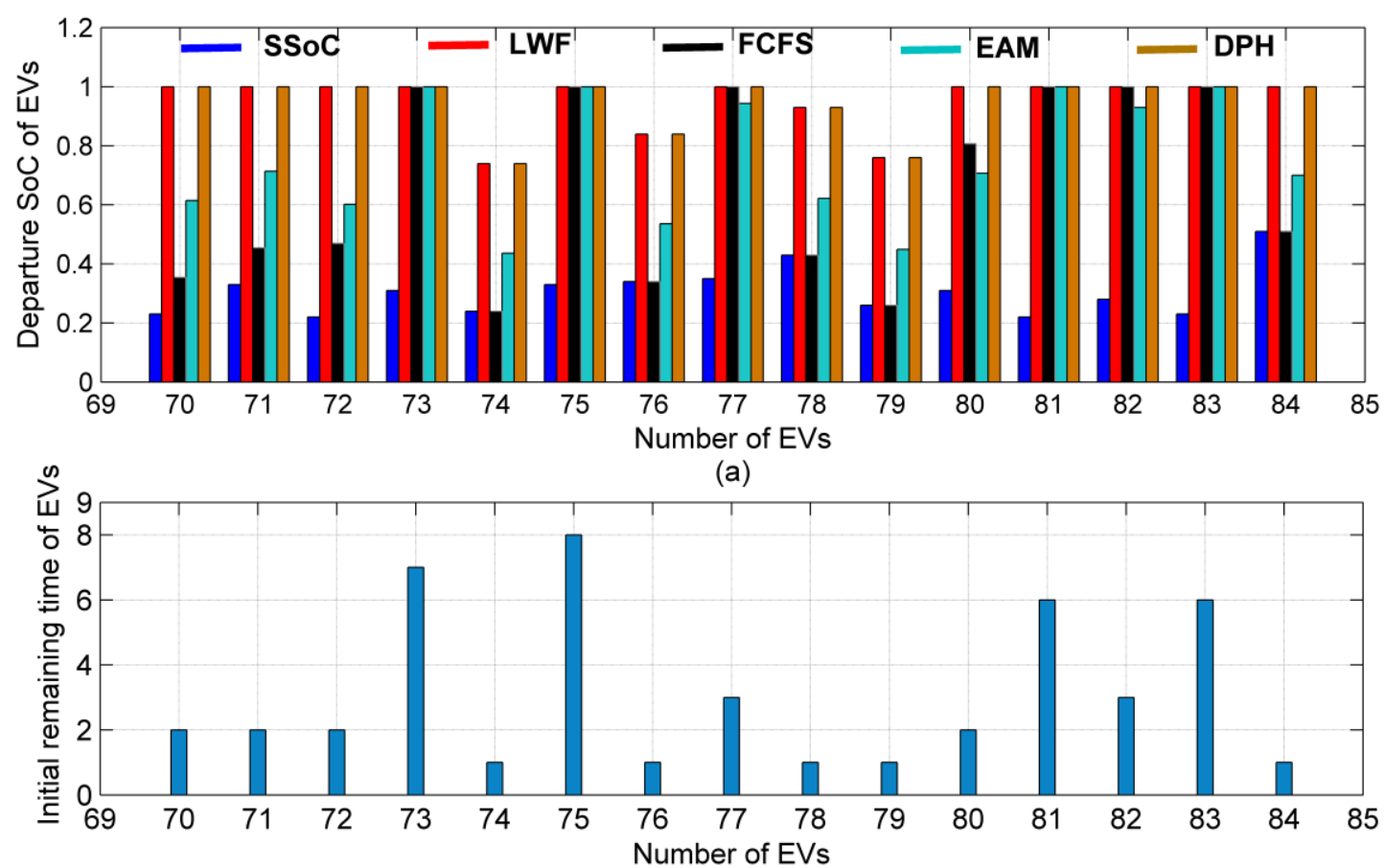

(b)

Figure 5. Comparison of the Satisfaction of Customer Demand Charging Methods

\section{Conclusion}

In this paper, a multi-period dynamic optimization model for charging service station operation for maximizing the total revenue over the planning horizon was proposed. By using the dynamic programming heuristic algorithm, this study basically solved the optimal power allocation to $\mathrm{PHEV} / \mathrm{EVs}$ problem for the charging station. The mathematical model for dynamic programming was built successfully. A heuristic algorithm for narrowing search space was proposed to solve the problem of large curse of dimensionality; this is beneficial as the dynamic programming is applied for a problem with a large number of PHEVs/EVs and period of time. The simulation results showed that the optimal power allocation to PHEVs/EVs with the DPH algorithm could solve the optimality between the revenue of the charging station and the departure $S o C$. Furthermore, the analysis in section 3 showed that the DPH algorithm can ensure a reasonable level of battery power attained for the vehicles with a short charging time, even in the event of an early departure.

\section{Acknowledgements}

The research presented in this paper is supported in part by the National Natural Science Foundation of China (61221063, U1301254, 61473218, 61304212).

\section{References}

[1] D. Lemoine, D. Kammen, and A. Farrell, "An innovation and policy agenda for commercially competitive plug-in hybrid electric vehicles", Environmental Research Letters, vol. 3, no.1, (2008), pp.014003.

[2] M. Duvall and E. Knipping, "Environmental Assessment of Plug-In Hybrid Electric Vehicles", EPRI (2007). Available at:

http://mydocs.epri.com/docs/CorporateDocuments/SectorPages/Portfolio/PDM/PHEV-ExecSum-vol1.pdf 
[3] S. Wirasingha, N. Schofield, and A. Emadi, "Plug-in hybrid electric vehicle developments in the US: Trends, barriers, and economic feasibility," Vehicle Power and Propulsion Conference, 2008. VPPC '08, IEEE, (2008) Sept 3-5, Harbin, pp. 1-8.

[4] K. Morrow, D. Karner, and J. Francfort, "Plug-in Hybrid Electric Vehicle Charging Infrastructure Review", U.S. Department of Energy Vehicle Technologies Program - Advanced Vehicle Testing Activity, Final Report Battelle Energy Alllance, (2008) November.

[5] W. Su and M-Y. Chow, "Sensitivity Analysis on Battery Modeling to Large-scale Charging Algorithms", IECON 2011 37th Annual Conference of the IEEE Industrial Electronics Society, (2011) Nov 7-10, Melbourne, Australia.

[6] P. Richardson, D. Flynn, and A. Keane, "Optimal Charging of Electric Vehicles in Low Voltage Distribution Systems", IEEE Transactions on Power Systems, vol. 27, no.1, (2012), pp. 268-279.

[7] W. Su and M-Y. Chow, "Performance Evaluation of An EDA-based Large-scale Plug-in Hybrid Electric Vehicle Charging Algorithm", IEEE Transactions on Smart Grid, vol. 3, no. 1, (2012), pp. 308-315.

[8] W. Su and M-Y. Chow, "Performance Evaluation of A PHEV Parking Station Using Particle Swarm Optimization”, IEEE Power and Energy Society General Meeting: 2011 IEEE, (2011) July 24-29, San Diego, pp. 1-6.

[9] W. Su and M-Y. Chow, "Computational Intelligence-based Energy Management for a Large-scale PHEV/PEV Enabled Municipal Parking Deck”, Applied Energy, vol. 96, (2012), pp. 171-182.

[10] I. Pahman, P. M. Vasant, B. S. M. Singh and M. Abdullah-Al-Wadud, "Intelligent energy allocation strategy for PHEV charging station using gravitational search algorithm", Proceedings of the $3^{\text {rd }}$ International Conference on Fundamental and Applied Sciences (ICFAS), (2014) June 3-5, Kuala Lumpur, Malaysia.

[11] Y. Cao, S. Tang, C. Li, P. Zhang, Y. Tan, Z. Zhang, and J. Li, "An optimized EV charging model considering TOU price and SOC curve", IEEE Transactions on Smart Grid, vol. 3, no. 1, (2011), pp. 388-393.

[12] D. H. Ban, G. Michailidis, and M. Devetsikiotis, "Demand Response Control for PHEV Charging Stations by Dynamic Price Adjustments", Innovative Smart Grid Technologies (ISGT), IEEE PES, (2012), Jan 16-20, Washington, DC, pp. 1-8.

[13] K. Young, C. Wang, L. Y. Wang and K. Strunz, "Electric vehicle battery technologies", Springer New York Publishers, New York (2012), pp. 15-56.

[14] R. A. Howard, "Dynamic Programming and Markov Processes”, John Wiley \& Sons, (1960)

[15] G. Hadley, "Nonlinear and Dynamic Programming", Addison-Wesley Publisher, (1964).
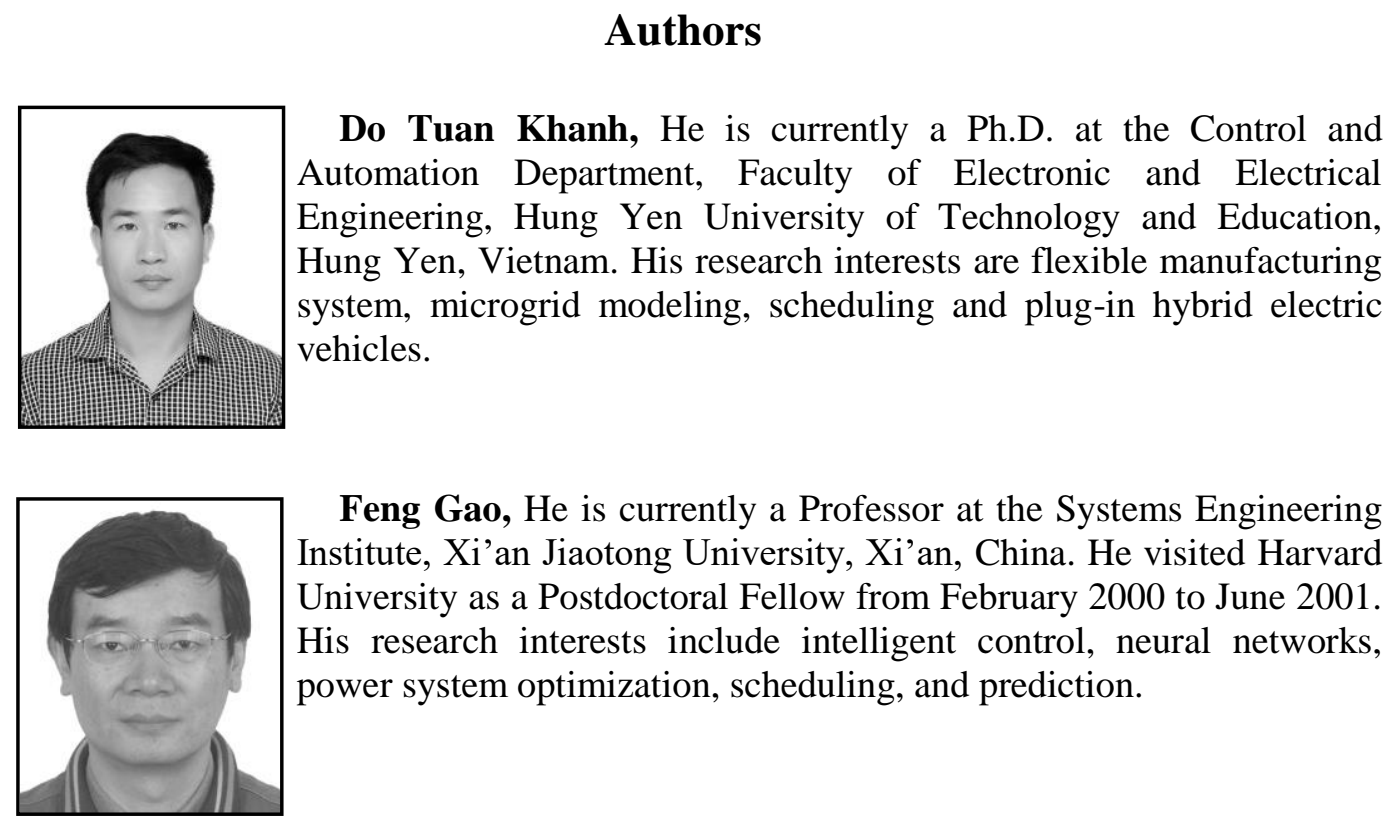

Feng Gao, He is currently a Professor at the Systems Engineering Institute, Xi' an Jiaotong University, Xi' an, China. He visited Harvard University as a Postdoctoral Fellow from February 2000 to June 2001. His research interests include intelligent control, neural networks, power system optimization, scheduling, and prediction. 